\title{
Ultrasonography of the pancreas, liver, and biliary system in cystic fibrosis
}

\author{
R C WILSON-SHARP, H C IRVING, R C BROWN, D M CHALMERS, \\ AND J M LITTLEWOOD \\ Departments of Radiology, Medicine, and Paediatrics, St James's University Hospital, Leeds
}

SUMmaRY Abdominal ultrasound imaging was performed in 50 children. Thirty nine were proved to have cystic fibrosis while 11 had respiratory infections and thus acted as controls. The pancreas was abnormal in $75 \%$ of cystic fibrosis patients aged under 5 years and in $95 \%$ over 5 years. In cystic fibrosis patients over 5 years old who had malabsorption, the pancreas was abnormal in $100 \%$. Abnormalities of the liver parenchyma were found in $23 \%$, of the gallbladder in $24 \%$, and splenomegaly in $8 \%$ of the cystic fibrosis patients.

Cystic fibrosis is the most common recessive inherited disease in Caucasians with an incidence of approximately 1:2500, and one in 20 of the population are carriers. In large centres experienced in the management of cystic fibrosis, $80 \%$ of patients now live beyond their 20th birthday, ${ }^{1}$ largely because of advances in the management of the respiratory complications. Increased survival has resulted in the growing importance of problems related to the dysfunction of the pancreas, liver, and biliary system. Detection of liver involvement in cystic fibrosis is notoriously difficult. Over inflation of the lungs makes radiological and clinical assessment of the liver and splenic size unreliable. Liver function tests do not become abnormal until relatively late in the disease and may not be seriously deranged even in the presence of oesophageal varices. Needle biopsy is of limited value in detecting the focal liver involvement. ${ }^{2}$ Ultrasound scanning can be used to detect and, by serial examinations, monitor the progress of liver changes and the signs of portal hypertension. Occasionally the diagnosis of cystic fibrosis is in doubt and the ultrasonic finding of a definitely abnormal pancreas can be helpful in supporting the diagnosis.

\section{Patients}

A total of 50 patients were scanned. Eleven were children with chest infections who acted as controls and 39 had typical cystic fibrosis with abnormal sweat electrolytes by the pilocarpine iontophoresis method. The age range was 1 to 21 years and 12 of the cystic fibrosis patients were under 5 years
Table 1 Age distribution of the 50 patients and controls scanned

\begin{tabular}{lrllll}
\hline \multicolumn{4}{l}{ Age (years) } \\
\cline { 2 - 5 } & $0-5$ & $5-10$ & $10-15$ & $15-21$ \\
\hline Controls $(\mathrm{n}=11)$ & 3 & 5 & 3 & 0 \\
Cystic fibrosis patients $(\mathrm{n}=39)$ & 12 & 8 & 16 & 3 \\
\hline
\end{tabular}

(Table 1). Nineteen of the cystic fibrosis patients were boys and 20 were girls.

\section{Methods}

A number of biochemical liver and pancreatic function tests were performed as part of a periodic comprehensive clinical and laboratory assessment of cystic fibrosis patients. Of particular relevance are determinations of serum bilirubin, serum alanine leucine transaminase, alkaline phosphatase, albumin, immunoreactive trypsin, prothrombin time, and the faecal fat output and percentage fat absorption. These results were compared with the ultrasonic findings.

The ultrasound scans were performed in a routine clinical department by a variety of ultrasonographers using a number of commercially produced machines. During the initial phase of the study static 'B' scanners were used, while later on real time scanners became available. The patients were fasted and neither the ultrasonographers nor the two radiologists who interpreted the scans (HCI and CWS) were aware of whether the patient had cystic fibrosis or was a control. The scanning techniques 

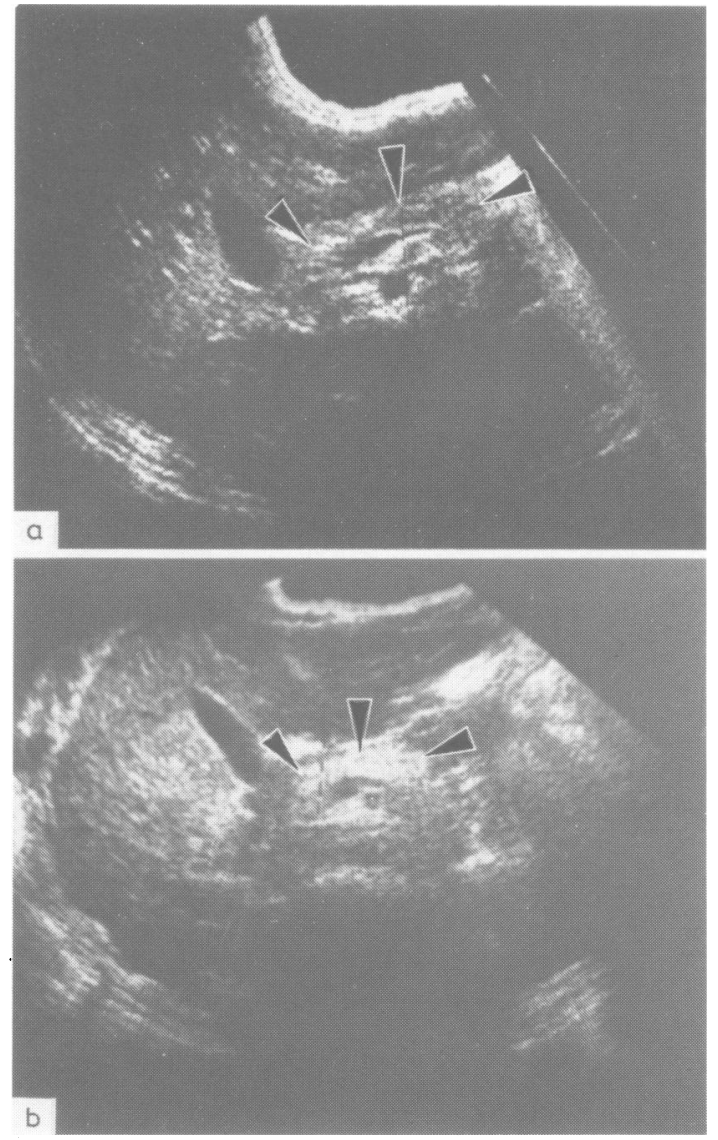

Fig. 1 (a) Transverse scan showing grade 1 abnormality of the pancreas (arrowheads) with echogenicity of greater amplitude than the liver, and (b) Transverse scan showing grade 2 abnormality of the pancreas (arrowheads) with noticeable increase in echogenicity and also shrinkage of the pancreas.

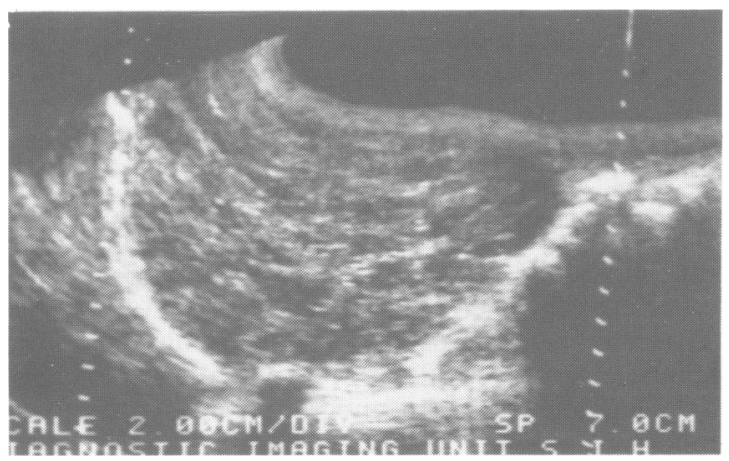

Fig. 2 Longitudinal scan through the right lobe of the liver showing patchily increased echogenicity. and criteria for scan interpretation used in our department have recently been described. ${ }^{3}$ Echo amplitude analysis was performed on a subjective basis using echo amplitude levels in other nonpathological organs for reference and taking into account other factors such as signal processing artefacts and transducer beam profile characteristics that would affect the recorded echo amplitudes.

The pancreas was considered normal if its echogenicity was approximately the same as the liver parenchyma at the same depth from the skin surface. The liver was first assessed by comparison with the parenchyma of the right kidney so that it could be used as a true reference. The pancreas was classified as abnormal if it was considerably more echogenic than the liver at the same depth, and was subjectively graded into 1 and 2 -grade 2 being the more echogenic (Fig. 1). Obvious shrinkage of the pancreas was noted, although absolute measurements were not recorded. Measurements can be unreliable since the shape of the pancreas varies with respiration and often the margins of the shrunken pancreas become indistinct and on ultrasound scan seem to merge with surrounding retroperitoneal fat. The liver was considered normal if it was only slightly more echogenic than the right renal cortex at an equivalent depth. Diffusely or patchily increased echogenicity was considered abnormal (Fig. 2). The appearances of the gallbladder were documented, and the presence or absence of stone or biliary sludge was noted. A subjective assessment of gallbladder size was made, and microgallbladder was diagnosed when obviously very small and shrunken (measurements of less than $3 \times 1 \mathrm{~cm}$ are considered indicative of microgallbladder) ${ }^{4}$

Spleen size was assessed by comparison with the left kidney but portal vein size was not documented because of the reported low sensitivity of calibre measurements in the detection of portal hypertension. 5

\section{Results}

The pancreas was normal in nine of the 11 control children and could not be visualised in two. Of 27 patients with cystic fibrosis in whom the pancreas was visualised, it was normal in three $(11 \%)$ but abnormal in $24(89 \%)$. The pancreas could not be visualised in 12 , mainly because of bowel gas which is a particular problem in cystic fibrosis patients (Table 2).

Two of the three patients with normal ultrasonic appearances of the pancreas were young (1.3 and 4.3 years) and it may be that the characteristic pancreatic changes of cystic fibrosis had not pro- 
Table 2 Results of pancreatic ultrasound in 50 patients and controls

\begin{tabular}{|c|c|c|c|c|}
\hline & \multicolumn{4}{|c|}{ Ultrasonic appearance of pancreas } \\
\hline & \multirow[t]{2}{*}{ Normal } & \multicolumn{2}{|c|}{ Abnormal } & \multirow[t]{2}{*}{ Not visualised } \\
\hline & & Grade 1 & Grade 2 & \\
\hline Controls $(n=11)$ & 9 & 0 & 0 & 2 \\
\hline $\begin{array}{l}\text { Cystic fibrosis } \\
\text { patients }(n=39)\end{array}$ & 3 & 3 & 21 & 12 \\
\hline
\end{tabular}

gressed to a sufficient degree. The third was older (12.2 years) but interestingly he was the only patient in our study without steatorrhoea. He represents one of the very few older patients who have no clinical evidence of an affected pancreas. ${ }^{6}$ Thus, the ultrasonic appearance of the pancreas was abnormal in six of eight $(75 \%)$ of the cystic fibrosis patients aged under 5 years in whom it was visualised. Over age 5 years it was abnormal in 18 of $19(95 \%)$. In patients aged over 5 years with malabsorption, however, the pancreas was abnormal in $100 \%$.

The liver was normal in all 11 controls but abnormal in eight of the 39 cystic fibrosis patients $(23 \%)$. Four had diffusely echogenic scans and five showed patchy echogenic areas in the periportal tracts (Table 3). One of the patients with an abnormal ultrasonic appearance of the liver had a liver biopsy which showed mild fatty infiltration and a focal biliary cirrhosis. This patient also had ultrasonic findings of grade 2 pancreatic involvement and splenomegaly.

The gallbladder was normal in all 11 controls but abnormal in eight of $33(24 \%)$ cystic fibrosis patients in whom it was visualised. One had a stone, two had biliary sludge, and five had a microgallbladder. The gallbladder was not visualised in six cystic fibrosis patients but it was impossible to say whether this was due to a severe degree of microgallbladder or to the obscuring effects of bowel gas (Table 4).

Splenomegaly was detected ultrasonically in three patients $(8 \%)$ with cystic fibrosis, all of whom had abnormal liver ultrasonic findings.

Liver function tests showed poor correlation with ultrasonic findings in the liver. Nine cystic fibrosis

Table 3 Results of liver ultrasound in 50 patients and controls

\begin{tabular}{lll}
\hline & \multicolumn{2}{l}{ Ultrasonic appearance of liver } \\
\cline { 2 - 3 } & Normal & Abnormal \\
\hline Controls $(\mathrm{n}=11)$ & 11 & 0 \\
Cystic fibrosis patients $(\mathrm{n}=39)$ & 30 & 9 \\
\hline
\end{tabular}

Table 4. Results of gallbladder ultrasound in 50 patients

\begin{tabular}{|c|c|c|c|c|c|}
\hline & \multicolumn{5}{|c|}{ Ultrasonic appearance of gallbladder } \\
\hline & \multirow[t]{2}{*}{ Normal } & \multicolumn{3}{|c|}{ Abnormal } & \multirow{2}{*}{$\begin{array}{l}\text { Not } \\
\text { visualised }\end{array}$} \\
\hline & & Stone & Sludge & Microgallbladder & \\
\hline $\begin{array}{l}\text { Controls }(n=11) \\
\text { Cystic fibrosis }\end{array}$ & 11 & 0 & 0 & 0 & 0 \\
\hline patients $(n=39)$ & 25 & 1 & 2 & 5 & 6 \\
\hline
\end{tabular}

patients had an abnormal serum alkaline phosphatase concentration and four of these had abnormal ultrasonic appearances. Only two of the nine patients with a raised serum alanine leucine transaminase concentration had abnormal ultrasonic findings. The two patients with grade 2 appearances in the liver both had abnormal alanine leucine transaminase and alkaline phosphatase concentrations. The serum bilirubin concentration was abnormal in only one patient: she had cystic fibrosis, an abnormal liver on ultrasound, and splenomegaly. The prothrombin time was abnormal in only two patients, both of whom had abnormal liver scans.

\section{Discussion}

Liver involvement in cystic fibrosis occurs in 20 to $50 \%$ of patients, depending on age and on what type of pathology is included. ' Focal or extensive biliary cirrhosis is the most common hepatic abnormality. The histological features are bile duct proliferation and ectasia secondary to obstruction by eosinophilic concretions, and periportal inflammatory cell infiltration. The appearances are specific for cystic fibrosis. In a few cases (approximately 5\%) this progresses and a multilobular biliary cirrhosis develops. Pathological examination at this stage shows large irregular nodules separated by massive foci of fibrosis but with areas of preserved architecture. Bile plugged ducts may be present but bile stasis within the lobules is conspicuously rare even in advanced liver disease. The age of onset of liver disease varies. It may present in the neonate but generally it presents later, the mean age of diagnosis being 8.7 years. ${ }^{7}$ It may be unrelated to the severity of disease elsewhere but generally there is a tendency for the liver disease to progress with age.

The poor correlation in cystic fibrosis between liver function and liver involvement is due to the focal nature of the liver disease which affects the biliary tracts so that a large volume of liver may be affected before the serum alkaline phosphatase and bilirubin concentrations rise. The serum alanine leucine transaminase determination, being a test of liver cell function, is likewise relatively unaffected. 
The serum albumin concentration has been shown to correlate better with the lung function than the liver disease in cystic fibrosis. ${ }^{8}$ The prothrombin time may be abnormal because of malabsorption and antibiotic treatment affecting the gut flora.

Only one of our patients had a liver biopsy. We did not consider it justifiable in the other children. In a previous study of liver biopsy in 84 cystic fibrosis patients the liver was affected in 15 $(17 \cdot 8 \%) .^{2}$ There was poor correlation between clinical, biochemical, and biopsy results. Biopsy correlation with liver ultrasound was reported by Willi et al in nine patients with cystic fibrosis who had patchy, irregular, or increased liver echogenicity. In all these the biopsy was abnormal. Ultrasound cannot reliably differentiate the different forms of liver involvement in cystic fibrosis (focal biliary cirrhosis, hepatitis, and cirrhosis) but it may be used as a screen in deciding which patients should undergo biopsy.

Portal hypertension develops in approximately two per cent ${ }^{110}$ of patients with cystic fibrosis. In our series we used only ultrasound assessment of spleen size to detect this complication, as explained previously. We do not currently have a combined Doppler B scanner which may be more useful in assessing portal hypertension and direction of flow within the portal veins. Rarely, gastric varices and portal venous collaterals may be detected on ultrasound.

Occasionally the diagnosis of cystic fibrosis is in doubt despite a meticulously performed sweat test, which may be equivocal or very rarely normal. Some of these patients undoubtedly have cystic fibrosis, and if the ultrasonic appearance of the pancreas is obviously abnormal this can be used as further evidence in supporting the diagnosis. Conversely a normal pancreatic appearance in a patient of 5 years or more with suspected cystic fibrosis is further evidence against the diagnosis. Our figures for cystic -fibrosis patients of $95 \%$ abnormal pancreatic ultra- sonic findings over 5 years and $100 \%$ abnormal over 5 years with malabsorption are useful in this respect.

We believe abdominal ultrasound is useful in the diagnosis, detection of complications, and follow up of cystic fibrosis patients and plan to scan all our patients at regular intervals. The technique has a place both in documenting the natural history of the condition and monitoring any modification of this that may occur as a result of future treatment.

\section{References}

I Park RW, Grand RJ. Gastrointestinal manifestations of cystic fibrosis: a review. Gastroenterology 1981;81:1143-61.

2 Dietzch HJ, Gottshalk B, Hinkel GK, Roschlau G. The value of bioptic follow-up examinations for controlling the course of liver involvement in cystic fibrosis. In: Sturgess SM, ed. Perspectives in cystic fibrosis. Toronto: Canadian Cystic Fibrosis Foundation, 1980:220-4.

${ }^{3}$ Irving $\mathrm{HC}$, Mitchell CJ. Inflammatory disease of the pancreas. Clinics in Diagnostic Ultrasound 1983;11:185-215.

4 Hubbard VS, Head GL, Shawker TH, et al. Radiological and ultrasound evaluation of the gallbladder in patients with cystic fibrosis. Pediatr Res 1978;12:437.

5 Bolondi L, Gardolfi L, Aneiti V, Caletti GC, Gaskarrini G, Labo G. Ultrasonography in the diagnosis of portal hypertension: diminished response of portal vessels to respiration. Radiology 1982;142:167-72.

' di Sant'Agnese PA. Davis PB. Cystic fibrosis in adults. Seventy five cases and a review of 232 cases in the literature. Am J Med 1979;66:121-32.

7 Psacharolpoulos HT, Howard ER, Portmann B, Mowat AP, Williams R. Hepatic complications of cystic fibrosis. Lancet 1981 ;ii:78-80.

\& Lykkegaard E, Jacobser L, Flensborg EW. Serum protein paper electrophoresis in cystic fibrosis correlated with clinical and radiological findings. Dan Med Bull 1974;21:232.

${ }^{9}$ Willi UV, Reddish JM, Littlewood Teele R. Cystic fibrosis: its characteristic appearance on abdominal sonography. Am J Radiol 1980;134:1005-10.

10 di Sant'Agnese PA, Blanc WA. A distinctive type of biliary cirrhosis of the liver associated with cystic fibrosis of the pancreas: recognition thro' signs of portal hypertension. Pediatrics 1956;18:387-409.

Correspondence to Dr H C Irving, Department of Radiology, St James's University Hospital, Beckett Street, Leeds LS9 7TF.

Received 4 May 1984 